\title{
Références bibliographiques du dossier « la formation tout au long de la vie »
}

\section{Marie-France Pamart}

\section{(2) OpenEdition}

12 Journals

Édition électronique

URL : http://journals.openedition.org/ries/3057

DOI : 10.4000/ries.3057

ISSN : 2261-4265

Éditeur

Centre international d'études pédagogiques

Édition imprimée

Date de publication : 1 décembre 1997

Pagination : 123-132

ISSN : 1254-4590

\section{Référence électronique}

Marie-France Pamart, « Références bibliographiques du dossier « la formation tout au long de la vie »

», Revue internationale d'éducation de Sèvres [En ligne], 16 | Décembre 1997, mis en ligne le 10 juin

2013, consulté le 03 mai 2019. URL : http://journals.openedition.org/ries/3057 ; DOI : 10.4000/

ries.3057

Ce document a été généré automatiquement le 3 mai 2019.

(c) Tous droits réservés 


\title{
Références bibliographiques du dossier "la formation tout au long de la vie"
}

\author{
Marie-France Pamart
}

1 Cette bibliographie sur le thème de la «formation tout au long de la vie » présente une sélection de documents (1994-1997) français et étrangers référencés dans la base de données du service de documentation du CIEP. Elle est organisée autour de quatre axes : approche conceptuelle, recommandations des organisations internationales, méthodologie de formation et, enfin, état des lieux en France et dans le monde.

\section{Approche conceptuelle}

MINISTÈRE DU TRAVAIL ET DES AFFAIRES SOCIALES, Se former tout au long de la vie, Entretiens Condorcet, sixièmes rencontres de la formation professionnelle, Paris, 30 septembre et $\mathrm{I}^{\mathrm{er}}$ octobre 1996, Paris, Le Monde/ministère du Travail et des Affaires sociales, 1997, $209 \mathrm{p}$.

Se former tout au long de la vie: ce n'est pas un hasard si le thème des Entretiens Condorcet en 1996 coïncidait avec celui de l'Année européenne ; l'éducation permanente est devenue une nécessité encore plus criante avec l'évolution de nos sociétés. Depuis la loi du 16 juillet 1971, la formation professionnelle continue a connu un développement constant et de plus en plus les entreprises la considèrent comme une condition de leur compétitivité; mais l'évolution incertaine de la notion de travail et de son lien avec l'emploi rend à la fois nécessaire, mais aussi particulièrement difficile, la conception d'un nouveau système de formation tout au long de la vie, fondé sur la valorisation des compétences et destiné à reconstruire la confiance face à des parcours professionnels de plus en plus hachés. Ce livre, issu des sixièmes Entretiens Condorcet sur la formation professionnelle, rend compte des débats qui agitent les meilleurs spécialistes français et européens de ce domaine.

MAUBANT Philippe (coord.), «L'alternance en formation, un projet à construire », Pour, n¹54, 1997/06, p. 5-177. 
La société attend beaucoup de la formation en alternance comme pouvant constituer un des points clés du système éducatif: celui du passage d'une démocratisation de la fréquentation scolaire à une démocratisation de la réussite scolaire. Dans ce numéro, praticiens et chercheurs se proposent d'analyser le concept d'alternance en formation sous différents angles, pédagogique, didactique, sociologique...

MONOD Ambroise (coord.), PERKER Henriette (coord.), "Se former tout au long de la vie", Actualité de la formation permanente, $n^{\circ} 141,1996 / 04$, p. 37-122, bibliogr.

Ce concept couvre maintenant tous les âges et tous les types d'apprentissage. Le dossier fait état de la diversité des conceptions et expériences, tant en ce qui concerne les formations liées au métier que celles qui sont destinées à améliorer la culture générale ou qu'on entreprend pour le plaisir d'apprendre.

5 AVANZINI Guy, L'éducation des adultes, Paris, Anthropos, "Exploration interculturelle et science sociale », 1996, XIV-182 p., bibliogr.

Après avoir clarifié la terminologie dans ce domaine, l'auteur s'interroge sur ce que signifie la notion d'éducabilité de l'adulte. 11 analyse ensuite les attentes et les caractéristiques de la population considérée, les conditions auxquelles une formation est efficace, le rôle et la responsabilité du formateur.

6 "Éducation/formation des adultes : question de terminologie", Langage et l'homme (Le), vol. XXXI, n 2-3, 1996/10, p. 189-202, bibliogr.

À l'occasion du lancement de l'Année européenne de « l'éducation et de la formation tout au long de la vie ", l'auteur cherche à préciser le sens de termes comme : éducation des adultes, éducation permanente, formation continue, en les comparant avec leurs homologues anglais. L'article se termine par un élément de lexique français-anglaisespagnol tournant autour de ces notions d'éducation/formation des adultes.

MÉHAUT Philippe, «Se former tout au long de la vie?», Bref/CEREQ, $n^{\circ}$ 120, 1996/05, p. 1-4.

La perspective européenne de «la formation tout au long de la vie » rencontre-t-elle la tradition française de la promotion sociale? Après avoir fait le diagnostic de cette interrogation, l'article étudie quelles sont les conditions à réunir pour construire une alternance tout au long de la vie.

8 VIRVILLE Michel de (rapp.), Donner un nouvel élan à la formation professionnelle, Rapport au ministre du Travail et des Affaires sociales, Paris, Documentation française, "Bilans et rapports », 1996, $148 \mathrm{p}$.

Ce texte formule, tant pour la formation initiale que continue, les propositions résultant des travaux d'une commission d'experts et d'une large consultation des parties concernées. En annexe, il fournit des éléments statistiques et juridiques sur la formation professionnelle et son financement.

\section{Recommandations des organisations internationales}

dans l'Union, Luxembourg, Office des publications officielles des Communautés européennes, 1997, $48 \mathrm{p}$.

Ce rapport qui fait suite à la recommandation du Conseil des ministres de l'Union européenne de 1993, est basé sur des rapports des États membres et des organisations des partenaires sociaux. Il offre le premier diagnostic complet de la formation professionnelle continue en Europe. Trois grands constats s'en dégagent : l'objectif d'une généralisation 
de l'accès à la formation continue à tous les travailleurs de l'Union est loin d'être atteint ; des écarts très importants subsistent entre les États membres et les différentes catégories d'entreprises et de travailleurs; les initiatives dans le domaine sont très nombreuses provenant aussi bien des pouvoirs publics que des partenaires sociaux ou des entreprises.

ORGANISATION DE COOPÉRATION ET DE DÉVELOPPEMENT ÉCONOMIQUE, Manuel pour élaborer de meilleures statistiques de la formation : conception, mesure, enquêtes, Paris, OCDE, 1997, $306 \mathrm{p}$.

L'importance du capital humain dans le développement des économies modernes est incontestable; cependant, la difficulté qu'il y a à définir et mesurer la formation de capital humain fait qu'il est mal aisé d'en déterminer les effets ou d'en évaluer l'ampleur. L'objectif premier de ce manuel est de contribuer à remédier à cette difficulté en fournissant des conseils pour la collecte de données sur la formation organisée dans le secteur privé et le secteur public.

HORN Jakob (dir.), BÉLANGER Paul (dix.), Institut de coopération internationale de la Confédération allemande pour l'éducation des adultes (IIZ/DVV)/Bonn, Institut de l'Unesco pour l'éducation (IUE/Hambourg), "Apprendre à l'âge adulte : une clé pour le XXI siècle », Documents de référence CONFINTEA V ( $5^{\mathrm{e}}$ conférence internationale sur l'éducation des adultes), Hambourg 14-18 Juillet 1997, Éducation des adultes et développement, $n^{\circ}$ spécial, 1997, 268 .

Cette livraison est une compilation des documents préparés à l'intention de la majorité des groupes thématiques participant à la cinquième conférence internationale sur l'éducation des adultes. La documentation est ainsi mise à la disposition de tous les participants à Hambourg pour leur permettre d'avoir, au préalable, une vue d'ensemble sur l'évolution du débat, avant et pendant les sessions.

MILLER Riel (rap.), Mesurer le capital humain : vers une comptabilité du savoir acquis, Paris, OCDE, 1996, $126 \mathrm{p}$.

Fruit d'une collaboration entre le comité de l'éducation et le comité de l'emploi, du travail et des affaires sociales de l'OCDE, le rapport a comme objet d'étude les investissements dans la formation continue et permanente. Il vise à cerner le lien entre le capital humain et les performances économiques, à repenser les systèmes d'information et de prise de décision en matière de ressources humaines. L'analyse fait appel à la recherche effectuée dans trois disciplines : l'économie, la comptabilité et l'éducation.

DELORS Jacques (coord.), L'éducation : un trésor est caché dedans, Rapport à l'Unesco de la Commission internationale sur l'éducation pour XXIe siècle, Paris, O. Jacob/UNESCO, 1996, 311 p.

La Commission internationale sur l'éducation pour le $\mathrm{XXI}^{\mathrm{e}}$ siècle a regroupé des personnalités venues d'horizons géographiques, culturels et professionnels variés. A la demande de l'Unesco, elle a réfléchi sur les défis à relever par l'éducation dans les années à venir et préparé suggestions et recommandations. Le rapport issu de ses travaux montre l'importance de l'éducation pour la compréhension du monde, la démocratie et le développement. Il évoque ensuite les objectifs de l'éducation et sa nécessaire continuité au long de la vie. Les orientations proposées concernent les différents niveaux d'enseignement, le rôle des enseignants, les choix politiques et la coopération internationale.

COMMISSION EUROPÉENNE, Livre vert Éducation-formation-recherche: les obstacles à la mobilité transnationale, Luxembourg, Office des publications officielles des Communautés européennes, 1996/10, COM (96) 462 final, $57 \mathrm{p}$.

Suivant les termes du traité de la Communauté européenne, l'action communautaire doit 
favoriser la mobilité en matière d'éducation, de formation et de recherche. C'est dans le cadre de cette mission que la Commission a élaboré un Livre vert sur la mobilité transnationale. Ce document recense les obstacles que celle-ci rencontre et propose à la réflexion et au débat des pistes d'action susceptibles de faire disparaître ces obstacles. En annexe, rappel des différents programmes européens dans les domaines de l'éducation, de la jeunesse, de la recherche, de la formation professionnelle, etc.

GASS J.R., Objectifs, structure et moyens de l'éducation et de la formation tout au long de la vie, Document de réflexion édité par la Commission européenne, Luxembourg, Office des publications officielles des Communautés européennes, 1996, Éducation, formation, jeunesse, 35 p. Le concept d'éducation et de formation tout au long de la vie n'implique pas nécessairement l'apparition d'un nouveau «système ». Il constitue plutôt une approche centrée sur les possibilités et les processus propres à chaque individu et admet simultanément que ces possibilités soient prises en charge par de nombreux acteurs de la société, à savoir non seulement les systèmes officiels d'éducation et de formation, mais également la famille, l'entreprise et les médias. Avec l'Année européenne de l'éducation et de la formation tout au long de la vie, l'Union européenne a cherché à contribuer à l'avènement d'une société cognitive en Europe, notamment par des débats institutionnels dont ce document est un des reflets.

COMMISSION EUROPÉENNE, Livre blanc sur l'éducation et la formation: Enseigner et apprendre - Vers la société cognitive, Luxembourg, Office des publications officielles des Communautés européennes, 1995, 107 p.

Après une description des enjeux et une analyse des évolutions, le Livre blanc envisage les actions à mener dans les domaines de l'éducation et de la formation. Des mesures doivent être mises en œuvre dès 1996 et viser à encourager l'acquisition de connaissances nouvelles, rapprocher l'école de l'entreprise, lutter contre l'exclusion, maîtriser trois langues européennes, traiter sur un plan égal l'investissement physique et l'investissement en formation. La version anglaise de ce document a été publiée en 1996.

BOGARD Gérald, "Éducation des adultes et mutations sociales: reconstruire l'éducation des adultes ", Actualité de la formation permanente, $n^{\circ} 129,1994 / 04, p .26-36$.

Depuis une trentaine d'année, l'éducation des adultes connaît dans tous les pays d'Europe une faveur accrue. Pour lutter contre les dysfonctionnements de nos sociétés et pour accompagner les mutations économiques, l'éducation s'est indéniablement renouvelée dans ses pratiques. Au gré des urgences locales et des partenariats qui peuvent se constituer, des réponses s'organisent, dessinant une figure nouvelle de l'éducation. C'est cette reconstruction que tente d'analyser l'article de Gérald Bogard, article qui est une contribution au projet du Conseil de l'Europe «Éducation des adultes et mutations sociales » conduit de 1988 à 1993.

\section{Méthodologie de formation}

18 MAROY Christian, «Les sources organisationnelles des différences pédagogiques en formation d'adultes ", Sciences de l'éducation pour l'ère nouvelle (Les), vol. 30, n 1, 1997/03, p. 5-21.

Les pratiques pédagogiques en formation d'adultes présentent des aspects hétérogènes qui sont dus le plus souvent à trois facteurs : les caractéristiques du public en formation, celles des formateurs et enfin les relations qu'une organisation de formation entretient avec son environnement. C'est ce troisième facteur que l'article développe, en s'inspirant de la sociologie des organisations anglo-saxonnes. 
19 PERKER Henriette, DECRESSAC Françoise, "Valider les savoirs et les savoir-faire: une nouvelle étape pour la formation continue ", Inffo flash, $n^{\circ} 475,1997 / 05,8 p$.

Dans un contexte de formation tout au long de la vie, la réforme du système de formation professionnelle nécessite que soit mis en place un système de validation des acquis de la formation continue et de l'expérience professionnelle. Ce dossier aide à faire progresser la réflexion sur cette question très actuelle.

MINISTÈRE DE L'ÉDUCATION NATIONALE DE L'ENSEIGNEMENT SUPÉRIEUR ET DE LA RECHERCHE, Éducation-Entreprises : 20 ans de partenariat réussi, 1996/11, 63 p.

Cette brochure dresse un panorama des actions de coopération et des échanges entre enseignement et monde professionnel. Ils s'instaurent à travers diverses activités : relation formation-emploi, construction et rénovation des diplômes et formations, information sur les métiers, stages en entreprise, formation continue, contrats de recherche.

21 «Formations ouvertes et multimédias ", Éducation permanente, $n^{\circ} 126$, p. 5-58 (supplément).

Le point de vue de spécialistes de la formation continue en France sur l'organisation et la logique (aménagement du territoire) de la formation à distance, ainsi que sur l'approche participative, la création d'outils multimédias, leur utilisation dans l'enseignement des langues.

"La fonction Morale dans les organisations éducatives et les entreprises", Recherche et formation, $n^{\circ} 22,1996 / 09$, p. 5-139.

Regroupant plusieurs articles, ce dossier vise à mettre en parallèle la fonction tutorale dans le monde de l'entreprise et dans celui de l'éducation. En effet, le tutorat est en passe d'apparaître comme une voie privilégiée de développement conjoint des systèmes de formation et des systèmes de travail. Le présent volume a été construit autour de trois entrées : une entrée analysant la notion même de tutorat, une entrée centrée sur les dynamiques d'acteurs qui participent à la fonction tutorale (professionnels de la formation, tuteurs et tutorés...), enfin une entrée plus pédagogique mettant l'accent sur l'identification d'outils, de pratiques ou d'expériences d'ingénierie.

LECLERCQ Véronique (coord.), "La formation de base des adultes ", Dossier Éducations, $n^{\circ} 8$, 1996/05, p. 14-59, bibliogr.

Ce type de formation, qui renvoie à un problème social, est un domaine délimité mais multiforme de la formation continue. Un secteur ingénierie et recherche découle de cette intervention éducative. La diversité des interventions constituant le dossier montre la multiplicité des approches et la richesse du thème.

SIMULA Pierre, "Les organismes de formation continue: pluralité des activités, diversité de gestion des personnels ", Bref/CEREQ, n 126, 1996/12, p. 1-4.

Ce numéro présente des données issues d'une enquête statistique nationale réalisée par le CEREQ en 1994 et qui portait sur un échantillon représentatif des organismes de formation continue les plus importants. Cette enquête révèle la diversité des formes institutionnelles de ces organismes ainsi que des modes de gestion de leur personnel.

BEDUWE Catherine, ESPINASSE Jean-Michel, "L'université et ses publics", Éducation et formations, $n^{\circ}$ 40, 1995/03, p. 33-46, tabl, graph.

L'enquête expérimentale menée en 1992 auprès d'étudiants montre qu'un tiers d'entre eux environ ne relève pas de la formation initiale. Il est important de mesurer la contribution des universités à la formation permanente et d'envisager les problèmes organisationnels et pédagogiques que peut poser l'hétérogénéité des publics étudiants. 
GAUTIER-MOULIN Patricia (coord.), "L'évaluation de la formation ", Actualité de la formation permanente, $n^{\circ} 138,1995 / 10, p .41-92$, bibliogr.

Ce dossier s'intéresse aux pratiques d'évaluation des organismes de formation continue, des consultants et des entreprises. L'ensemble des témoignages présentés montre la diversification, le renouvellement et l'adaptation aux besoins des pratiques d'évaluation.

ASSOCIATION POUR LE DÉVELOPPEMENT ET L'INSERTION DES TECHNOLOGIES ÉDUCATIVES (ADITE), "De l'organisation formatrice à l'organisation apprenante», FIT FormationInformatique-Technologie, $n^{\circ}$ spécial, Paris, ADITE, 1995/06, $91 \mathrm{p}$.

Ce numéro spécial s'inscrit dans le cadre des réflexions menées par les groupes de travail de l'ADITE sur l'évolution des pratiques pédagogiques et des dispositifs de formation; à travers un certain nombre de témoignages venus du monde de la grande entreprise, les intervenants ont tenté d'expliciter des termes relativement nouveaux en France comme : organisation formatrice, organisation apprenante, organisation qualifiante.

LEPLATRE Françoise (coord.), "Qualité de la formation », Actualité de la formation permanente , $n^{\circ}$ 133, 1994/12, p. 57-112.

Ce dossier fait le point sur les répercussions qu'ont pu avoir, dans le secteur de la formation continue, les nouvelles approches de la qualité. Dans quelle mesure les organismes de formation ont-ils été influencés par les notions de certification, label, qualification? Même si la plupart des pratiques n'ont pas encore changé, les conceptions de la formation ont évolué, amenant les organismes à s'interroger sur leur organisation et leur pédagogie.

PREVOST Hervé, L'individualisation de la formation: autonomie et/ou socialisation, Lyon, Chronique sociale, 1994, $178 \mathrm{p}$.

L'auteur montre l'intérêt d'une individualisation de la formation des adultes comme facteur d'autonomisation et de socialisation. En analysant divers dispositifs de formation allant dans ce sens, il identifie les niveaux et les pratiques d'individualisation et montre qu'un bilan de celle-ci dans les institutions formatrices est nécessaire pour en évaluer les effets.

\section{État des lieux en France et dans le monde}

\section{Monographies}

CENTRE POUR LE DÉVELOPPEMENT DE L'INFORMATION SUR LA FORMATION CONTINUE, Les fiches pratiques de la formation continue, Paris-La Défense, Centre inffo, 1997, éd. 97, 960 p. + fasc. de mise à jour.

L'ouvrage présente l'ensemble du dispositif réglementaire de formation professionnelle continue en France. Les fiches et chapitres sont regroupés en deux parties: mesures d'accès à la formation ; interventions des acteurs de la formation. Des bulletins mensuels de mise à jour actualisent les données.

ANCEL François, «La formation des adultes réalisée dans les GRETA de l'Éducation nationale; bilan 1995 ", Note d'information - DEP, $n^{\circ}$ 97.03, 1997/01, 5 .

ESQUIEU Paul (éd.), «Formation professionnelle et enseignement technologique ", Éducation et formations, $n^{\circ} 45,1996 / 03$, p. 1-138.

Les articles rassemblés, en donnant la parole aux différents acteurs de ce secteur d'enseignement en France, dressent un large état des lieux. Historique et évolution, 
formation initiale et continue, diplômes, types d'établissements, voie technologique, apprentissage, enseignants, emploi et insertion des jeunes, tels sont les différents thèmes abordés.

BECCARELLI Catherine (coord,), MONOD Ambroise (coord.), « Région et formation », Actualité de la formation permanente, $n^{\circ} 139,1995 / 12, p .49-145$, bibliogr.

Après une analyse historique du texte de 1983 sur la décentralisation de la formation professionnelle, ce dossier présente le nouveau dispositif juridique, puis les partenariats État-régions et la mise en place des plans régionaux de développement des formations professionnelles des jeunes. Plusieurs régions présentent ensuite certaines réalisations. Une bibliographie et une liste d'adresses permettent d'approfondir le thème.

FORESTIER Christian, JEANTEUR Jacques, ZIGNOL Maurice, BRIDOUX Edouard, « Les GRETA ont 20 ans : 1974-1994 ", Éducation économie, $n^{\circ} 23,1994 / 07$, p. 39-48

Afin de célébrer les vingt ans des groupements d'établissements pour la formation continue des adultes, le dossier décrit l'histoire et les missions de ce service public, la politique et les actions menées par le ministère de l'Éducation nationale, l'organisation du réseau et ses liens avec les collectivités territoriales et les entreprises.

GAUTIER-MOULIN Patricia, "La formation en Islande : un système entièrement modulaire ", Inffo

flash, $n^{\circ}$ 465, 1996/11, p. 11-14.

Ce dossier fait le point d'une manière à la fois concise et précise sur le système de formation en Islande. Celui-ci se caractérise essentiellement par une organisation modulaire de toutes les formations, une grande perméabilité entre formation initiale et formation continue avec les mêmes enseignants et les mêmes programmes et une large utilisation des nouvelles technologies en formation.

MELIS Africa (coord.), CEDEFOP (Centre européen pour le développement de la formation professionnelle, Thessalonique), Enseignants et formateurs de formation professionnelle; vol. 1: Allemagne, Espagne, France et Royaume- Uni; vol. 2: Italie, Irlande, Portugal, Luxembourg, Office des publications officielles des Communautés européennes, 1996, $1^{\text {re édition, }}$ $207 p .+147 p$.

L'objectif de ces deux ouvrages est d'apporter une contribution à la connaissance mutuelle de la formation professionnelle dispensée dans les différents pays de l'Union européenne en décrivant les différents programmes; il se concentre sur un groupe important de professionnels qui en sont les véritables acteurs: les enseignants et les formateurs.

C'est à eux que s'adresse en premier lieu ce travail qui veut être un instrument pratique pour faciliter, en autre, leur mobilité à l'intérieur de l'Union européenne.

PARADIS Mylène, "La formation professionnelle au Québec: portrait d'un secteur en pleine mutation », Inffo flash, $n^{\circ} 456,1996 / 07$, p. 13-20.

Le mini-dossier décrit le système d'enseignement québécois. On note la volonté du gouvernement de resserrer les liens entre éducation et monde du travail, d'une part, et, d'autre part, d'ajuster les moyens afin de lutter contre le chômage et développer l'emploi.

Pour un accès réel des adultes à la formation continue, Avis à la ministre de l'Éducation, Sainte-Foy (Québec), Conseil supérieur de l'éducation, 1996, VIII-119 p.

Après avoir analysé les réalités sociales et les besoins de formation continue au Québec, le document présente les services et activités de formation des adultes dans le réseau scolaire et extra-scolaire. Il constate leur richesse et leur diversité, mais aussi leurs 
difficultés, et recommande des orientations et dispositifs à promouvoir pour améliorer le système de formation continue.

QUENOLLE Mirjam, PERKER Henriette, «Le système de formation en Finlande », Inffo flash, $n$ 427, 1995/04, p. 13-16, bibliogr.

La synthèse sur le système d'enseignement en Finlande s'intéresse à la formation initiale et à la formation des adultes et étend le sujet vers la coopération internationale. Bibliographie et adresses utiles.

BERT Claudie, «La formation des adultes en Israël ", Inffo flash, $n^{\circ}$ 436-437, 1995/08, p. 13-16, bibliogr.

En Israël, l'État incite les adultes à se former. Le dossier donne le schéma du système éducatif israélien et relève les particularités d'une politique marquée par le souci d'intégration et d'accueil des immigrants juifs et le rôle de l'armée.

41 MERLLIE Christine, «La formation professionnelle aux États-Unis », Inffo flash, $n^{\circ} 406,1994 / 04$, p. 11-14, bibliogr.

La synthèse sur la formation professionnelle aux États-Unis décrit le système éducatif, illustré d'un organigramme, ainsi que les conditions de formation des adultes. Une bibliographie et des adresses utiles complètent l'ensemble.

On trouvera également des données intéressantes dans les monographies réalisées par le CEDEFOP: "Le système de formation professionnelle au/en ». Ces études ont été préparées dans chacun des États membres de la Communauté en suivant des stipulations détaillées élaborées par le CEDEFOP quant au contenu demandé. Les derniers titres parus concernent l'Italie, le Portugal, le Danemark, le Luxembourg et la République fédérale d'Allemagne.

DOHMEN Günther, Lifelong Learning. Guidelines for a modem éducation policy, Federal Ministry of Education, Science, Research and Technology, (En anglais, original en allemand), Bonn, 1996, 317 p., bibliogr.

Cet ouvrage commandité par le ministère de l'Éducation, de la Science, de la Recherche et de la Technologie allemand examine les approches de la «formation tout au long de la vie ", telles qu'elles existent en Allemagne, mais aussi dans d'autres pays. L'étude indique des pistes concrètes d'action pour un dépassement des pratiques éducatives traditionnelles et une modernisation des politiques éducatives fondées sur une meilleure prise en compte des apprentissages individuels.

MINISTÈRE DE L'ÉDUCATION GÉNÉRALE ET PROFESSIONNELLE DE LA FÉDÉRATION DE RUSSIE, département de la formation professionnelle continue et académie d'État de l'innovation (Coll. sous la direction de S.V.Kondratieva), Recueil des textes réglementaires et des documents méthodologiques pour la formation professionnelle continue (en russe), Moscou, 1996, $289 \mathrm{p}$.

Cette publication officielle se situe dans le cadre de la loi de l'éducation du 13 janvier 1996 de Russie rappelée en début d'ouvrage et précise les conditions de l'organisation de la formation des professionnels adultes, qu'il s'agisse d'élévation des qualifications ou de reconversion. L'ensemble a été profondément rénové pour répondre aux nouvelles conditions économiques et sociales du pays et soutenir l'effort de développement de ressources humaines adaptées à la nouvelle donne. Outre les textes fondateurs du système, des documents et des recommandations méthodologiques permettent de comprendre les instruments à disposition des organismes prestataires de formation, publics et privés. 


\section{Études comparatives}

\section{Défense/Centre INFFO, 1996, Dossier documentaire, $62 \mathrm{p}$.}

Ce document constitué de fiches publiées à l'origine dans Inffo-Flash et mises à jour, offre un panorama de la formation professionnelle dans les pays de l'Union européenne (sauf la France) en distinguant quatre catégories de dispositifs en terme de formation continue: soit l'entreprise est au centre du premier concept de formation continue (Allemagne, Grande-Bretagne, Belgique, Autriche et Pays-Bas); soit ce sont les pouvoirs publics (Espagne, Portugal, Grèce, Danemark) ou la région (Italie). La dernière catégorie, fondée sur la loi et le droit, est représentée par le système français.

SKINNINGSRUD Tone, « Further Education and Training: a Comparison of Policy Models in Britain and Norway ", Comparative Education, vol. 31, $n^{\circ} 1,995 / 03$, p. 69-82, bibliogr.

Cet article établit une comparaison entre les systèmes de formation continue en Angleterre et en Norvège, tandis que le premier se plie à l'économie de marché, le second semble s'appuyer sur un modèle mixte, combinant le libéralisme avec un certain esprit collectiviste et dans une perspective d'intégration sociale.

ARNOVE Robert F., TORRES Carlos Alberto, «Adult Education and State Policy in Latin America: the Contrasting Cases of Mexico and Nicaragua ", Comparative Education, vol. 31, $n^{\circ} 3,1995 / 11$, p. 311-325, bibliogr.

Les auteurs ont choisi d'étudier deux modèles contrastés d'éducation des adultes en Amérique latine : celui du Mexique et celui du Nicaragua. Au Mexique, la formation des adultes repose sur le corporatisme et les normes de productivité tandis qu'au Nicaragua l'appréhension du problème est plus révolutionnaire. En dépit de ces deux approches idéologiquement différentes, les deux pays présentent des points communs, caractéristiques des pays d'Amérique latine sous l'influence de leur puissant voisin : les États-Unis. Avant d'étudier ces deux cas, les auteurs font un tour d'horizon de l'analphabétisme et de l'éducation des adultes en Amérique latine.

PERKER Henriette, "Se former tout au long de la vie en Europe», Inffo flash, $n^{\circ} 433,1995 / 07$, p. 13-16, bibliogr.

Dans la perspective de l'Année européenne de l'éducation et de la formation tout au long de la vie, ce dossier d'Inffo flash fait le point sur les principaux dispositifs des pays de l'Union en matière de formation continue.

48 "Recherche en éducation des adultes dans les années quatre-vingt-dix: que savons-nous? où allons-nous?", Éducation des adultes et développement, $n^{\circ} 45,1995$, p. 3-222, bibliogr.

Ce numéro spécial reprend certains extraits des rapports régionaux et d'autres articles préparés pour le séminaire international sur les «Tendances de la recherche en éducation des adultes ", qui s'est tenu à Montréal fin 1994. Il fournit ainsi un aperçu concernant aussi bien l'Afrique que l'Asie, l'Amérique ou l'Europe.

MONOD Ambroise (coord.), PERKER Henriette (coord.), "Éducation - formation - insertion en France et en Europe ", Actualité de la formation permanente, $n^{\circ} 129$, 1994/04, p. 25-103, bibliogr. Il est indispensable d'« éduquer les adultes pour combattre l'exclusion et gouverner les mutations sociales ». Défini par le Conseil de l'Europe, auquel sont empruntées certaines contributions de ce dossier, cet objectif apparait en filigrane dans les différents articles. Ceux-ci abordent les questions de la formation des adultes et de l'intégration sociale et 
professionnelle des populations défavorisées. Les relations d'expériences menées dans différents pays d'Europe proposent des amorces de réponses aux problèmes qui se posent. BELANGER Paul (ed.), GELPI Ettore (ed.), "Lifelong Education: Special Issue », International Review of Education, vol. 40, n³-5, 1994, p. 190-398, bibliogr.

L'idée de départ, à l'origine de ce numéro, est que l'éducation et l'éducation permanente, de plus en plus, ne font qu'un, et qu'il importe de ce point de vue d'étudier l'évolution des politiques et des pratiques éducatives. Ainsi sont analysées les politiques d'éducation permanente d'un certain nombre de pays comme l'Algérie, l'Argentine, le Bénin, le Chili, la Chine, le Japon, la Pologne et la Suède.

INDEX

Mots-clés : formation tout au long de la vie, compétence, formation alternée, formation continue, formation initiale, formation professionnelle, politique éducative, réforme de l'enseignement

\section{AUTEUR}

MARIE-FRANCE PAMART

Service de documentation, CIEP, France 